BNL-64989

\title{
ELECTRON DYNAMICS IN THE STRONG FIELD LIMIT OF PHOTOIONIZATION
}

RECEIVED

DEC 181997

O S.T

\author{
B Sheehy, ${ }^{1}$ B Walker, ${ }^{1} \mathrm{R}$ Lafon, ${ }^{1} \mathrm{M}$ Widmer, ${ }^{1} \mathrm{~L}$ F DiMauro, ${ }^{1} \mathrm{P}$ \\ Agostini, ${ }^{2}$ and K C Kulander, ${ }^{3}$ \\ ${ }^{1}$ Brookhaven National Laboratory, Department of Chemistry, Upton, \\ NY 11973 \\ ${ }^{2}$ Service des Photons, Atomes et Molécules, Centre d'Etudes de Saclay, \\ 91191 Gif Sur Yvette, France \\ ${ }^{3}$ TAMP Group, Lawrence Livermore National Laboratory, Livermore, \\ CA 94551
}

DSTRABUTON OF THIS DOCUAERT IS UNCHTED

High precision photoelectron energy and angular distributions in helium and neon atoms for a broad intensity range reflect the change in the continuum dynamics that occurs as the ionization process evolves into the pure tunneling regime. Elastic rescattering of the laser-driven free electron from its parent ion core leaves a distinct signature on the spectra, providing a direct quantitative test of the various theories of strong field multiphoton ionization. We show that it takes a relatively complete semi-classical rescattering model to accurately reproduce the observed photoelectron distributions. However, the calculated inelastic rescattering rate fails to reproduce the measured nonsequential double ionization yields.

\section{INTRODUCTION}

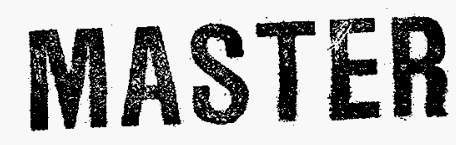

Strong field photoelectron spectra have attracted considerable attention over the past decade and a half, but a comprehensive understanding of the underlying dynamics which produce these spectra and related phenomena has solidified with the development of high repetition-rate, short pulse lasers which can span the entire intensity range of importance ${ }^{1}$. In $1965, \mathrm{Keldysh}^{2}$ showed that at infrared and visible wavelengths the dynamics of strong field atomic ionization undergoes a change in character as the laser intensity increases. In weak fields electrons are promoted into the continuum by the simultaneous absorption of enough photons to increase their energy above the ionization potential. This is called multiphoton ionization (MPI). However, as the laser intensity increases, a completely separate mode of escape becomes possible. At large distances from the nucleus the electrostatic attraction of 


\section{DISCLATMER}

Portions of this doecment may be illegible in electronic irosge products. Imoges are produced from the best available original docementert 


\section{DISCLAIMER}

This report was prepared as an account of work sponsored by an agency of the United States Government. Neither the United States Government nor any agency thereof, nor any of their employees, makes any warranty, express or implied, or assumes any legal liability or responsibility for the accuracy, completeness, or usefulness of any information, apparatus, product, or process disclosed, or represents that its use would not infringe privately owned rights. Reference herein to any specific commercial product, process, or service by trade name, trademark, manufacturer, or otherwise does not necessarily constitute or imply its endorsement, recommendation, or favoring by the United States Government or any agency thereof. The views and opinions of authors expressed herein do not necessarily state or reflect those of the United States Government or any agency thereof. 
the ion core can be overwhelmed by the laser's instantaneous electric field, producing a barrier through which a valence electron can tunnel. In this regime a quasi-static tunneling picture becomes appropriate: the laser field varies so slowly compared to the response time of the electron that the ionization rate becomes simply the cycleaverage of the instantaneous dc-tunneling rate. Tunneling becomes important when the ratio of the frequency of the applied field to the tunneling rate becomes smaller than unity. This ratio, known as the Keldysh or adiabaticity parameter, $\gamma$, is given by $\left[I_{p} /\left(2 U_{p}\right)\right]^{1 / 2}$, where $I_{p}$ is the binding energy of the electron and $U_{p}=2 \pi \alpha I / \omega^{2}$ is the ponderomotive energy (in atomic units) of a free electron in the laser field of frequency $\omega$ and intensity $I$, where $\alpha$ is the fine structure constant.

The majority of experimental studies on neutral atoms exposed to intense, shortpulse laser fields have been carried out in the MPI regime $(\gamma \geq 1)$. A few experiments ${ }^{3,4}$ have extended into the tunneling regime, but these measurements have been limited to observations of total ionization rates or electron energy distributions over a small dynamic range. In this paper, we report upon the first systematic experimental investigation in the strong field tunneling limit. Our study, by virtue of the enhanced dynamic range accessible with kilohertz laser technology, follows the change in the ion yield curves, electron spectrum and angular distributions as the ionization evolves from predominantly MPI to pure tunneling. The study examines both helium and neon atoms which have been established to tunnel ionize near the saturation intensity ${ }^{5}$. We find that the electron distributions in the tunneling regime are very different from any previous reports obtained in the MPI or mixed regimes ${ }^{6,7,8}$. We achieve a quantitative description of the electron spectra using a rescattering picture $e^{8,9}$ which mimics the time evolution of a tunnel-ionized continuum wave packet in the combined fields of the laser and ion core. However, when the same model is applied to the $\mathrm{e}-2 \mathrm{e}$ inelastic rescattering process, it fails, both quantitatively and qualitatively, to describe the measured nonsequential production of doubly charged helium and neon ions. Since rescattering events are known to be important in other short-pulse, strong-field emission phenomena (e.g., harmonic generation) this investigation better defines the underlying dynamics of these processes.

\section{QUASICLASSICAL MODEL IN THE TUNNELING LIMIT}

The motion of the tunneling wave packet is controlled mostly by its interaction with the laser field, since it rapidly moves beyond the range where the ion core potential is effective. Its evolution can be reasonably approximated using the classical equations of motion for a laser-driven electron. In a simple quasiclassical (SQC) model $^{3}$, the bound electron becomes free at a particular phase, $\omega t_{o}$, of the field, with zero kinetic energy, then undergoes oscillatory motion at the laser frequency, $\omega$. The time dependent electron velocity is given in atomic units by $v(t)=(\mathcal{E} / \omega) \times(\cos \omega t-$ $\left.\cos \omega t_{o}\right)$, and the kinetic energy by $T=2 U_{p} \times\left(\cos \omega t-\cos \omega t_{o}\right)$, where $\mathcal{E}=(8 \pi \alpha I)^{1 / 2}$ is the amplitude of the laser's electric field. The first term represents the electron's quiver motion: the time-averaged velocity is zero, while the average energy is $U_{p}$. The second, or drift, term represents the energy which would be detected in a short-pulse experiment. In the absence of further interactions with the ion core (rescattering) the maximum drift energy an electron can have is $2 U_{p}$.

The rescattering picture ${ }^{8,9}$ goes beyond the SQC model by recognizing that roughly half the electrons will recross the plane of the nucleus when the field has changed sign. We extend that model here to quantitatively model the photoelectron energy spectra (PES) in terms of elastic rescattering, and examine the model's 
potential for modeling multiple ionization as an inelastic rescattering process.

Only the first return to the core is considered. Due to dispersion of the tunneling electron wavepacket, the probability of rescattering on subsequent returns is negligible. We divide the optical cycle into a large number of equal time intervals. In each interval a trajectory is launched at the outer turning point of the suppressed effective potential with zero velocity. It is propagated in the combined fields of the laser and the helium (neon) ion core until either it escapes or returns to cross the plane of the nucleus. Those which escape contribute to the spectrum below $2 U_{p}$ according to their drift velocities, as in the SQC model. The returning trajectories are assumed to be guiding a freely spreading gaussian wave packet whose width is given by $\alpha(\tau)=\left(\alpha(0)^{2}+[2 \tau / \alpha(0)]^{2}\right)^{1 / 2}$, where $\alpha(0)$ is the initial width and $\tau$ is the propagation time between initiation and return. Choosing $\alpha(0)=4.0 a_{0}$, gives a return width consistent with our numerical studies ${ }^{5}$. We calculate the differential elastic scattering cross section for this wave packet using ${ }^{11}$

$$
\sigma(\theta)=\left|\frac{1}{2 i k} \sum_{\ell=1}^{\ell_{\max }} a_{\ell}(2 \ell+1) e^{2 i\left(\eta_{\ell}+\delta_{\ell}\right)} P_{\ell}(\cos \theta)\right|^{2} .
$$

Here $\delta_{\ell}$ is the Coulomb phase shift (for a charge of 1) and $\eta_{\ell}$ is the additional phase shift resulting from the short range part of the $\mathrm{He}^{+}\left(\mathrm{Ne}^{+}\right)$potential. These phase shifts are obtained from numerical integration of the scattering equations for electron$\mathrm{He}^{+}\left(\mathrm{Ne}^{+}\right)$over the necessary range of energies and angular momenta. The partial wave amplitudes, $a_{\ell}$ are determined from the distribution of impact parameters in the returning wave packet $\left(\ell=m v_{\text {ret }} b\right)$.

Equation (1) gives the field-free differential cross section. The laser field will distort this distribution. The transverse component of the outgoing velocity is conserved, but the velocity along the polarization direction has both a drift component and a quiver velocity which depend on the phase of the laser field at the return time. Drift velocities corresponding to $\mathrm{PE}$ energies as high as $10 U_{p}$ can be produced if the trajectory is scattered by $\sim 180^{\circ}$. The energy and angular distributions from the wave packet in each time interval are weighted by the instantaneous tunneling rate. In these calculations we have actually used a scaled dc-tunneling rate which, when cycle averaged, gives the ADK rate ${ }^{12}$. This accounts for the initial state not being purely hydrogenic. The total angle-resolved electron distribution for a given laser intensity is obtained by summing the contributions from all time intervals. The e-2e inelastic process, which leads to the production of double ionization, is calculated using a modified Lotz cross section ${ }^{13}$ which accounts for both excitation and ionization. Spatial and temporal averaging is performed for comparison to the experimental measurements.

\section{TUNNELING PHOTOELECTRON DISTRIBUTIONS}

In the experiments presented here a $120 \mathrm{fs}, 1 \mathrm{kHz}$ repetition rate, titanium sapphire laser operating at $0.78 \mu \mathrm{m}$ was focused by $\mathrm{f} / 4$ optics into an ultrahigh vacuum chamber containing helium or neon. Under these conditions, the system is capable of producing a maximum intensity of $20 \mathrm{PW} / \mathrm{cm}^{2}$ with typical pulse-to-pulse fluctuations $<1.7 \%$ for $10^{6}$ laser shots. Electrons or ions are analyzed using a $30 \mathrm{~cm}$ time-of-flight spectrometer. The laser polarization is $>99 \%$ over the entire intensity range, and is rotated relative to the detector axis to study angular distributions.

Total PES are shown in Fig. 1 for three different intensities in both absolute and ponderomotive energy units. A single experimental run measures the PES for one 
polar angle, and these distributions are constructed by integrating the angle-resolved PES (ARPES) over the polar: angle and assuming azimuthal symmetry around the polarization axis. The inset in Fig. la shows typical angular distributions at several energies.

The preponderance of photoelectron energies below $2 U_{p}$ is evident, consistent with the simple quasi-classical (SQC) model $^{3}$. The angular distributions of these electrons are strongly aligned along the laser polarization direction, becoming narrower towards the $2 U_{p}$ limit. However, a striking change occurs above $2 U_{p}$ : there the ADs are significantly broader (with a weak narrowing as the energy increases towards $10 U_{p}$ ), indicative of rescattering. In fact, this PES seems to be a superposition of two components: a "normal" narrow distribution that falls off rapidly with increasing energy between 0 and $2 U_{p}$ and a much broader but weaker, almost flat energy distribution that extends out to high energies before abruptly truncating at $8-10 U_{p}$. As the intensity increases, moving further into the tunneling regime, the fraction of electrons in this high-energy portion falls as $I^{-2.5}$. These results differ dramatically from all previous experimental reports ${ }^{1}$. For example, the PES for inert gas atoms ${ }^{6,7}$ clearly show angle-dependent structures, as well as an abundance of electrons with energies $>2 U_{p}$. These differences reflect the pure tunneling nature in these experiments and provides a unique opportunity for quantitatively testing the rescattering picture.

The quantitative comparisons of the measured and calculated total (spatially and temporally averaged) PES are also shown in Fig. 1. The dashed-dotted line in Fig. 1(b) represents the SQC model. The obvious failure of the model in predicting the high energy portion of the spectrum indicates the necessity of including rescattering. The dashed lines are the calculated PES using the $\mathrm{He}^{+}$potential and give excellent agreement with the experimental measurement over the entire energy range for both "pure" tunneling cases. The poorer agreement seen in Fig. 1(c) signifies the transition into the mixed regime where the MPI contribution is becoming significant. Figure 1(b) also shows a PES calculated using a pure hydrogenic potential (dotted line). Coulomb scattering significantly underestimates the high energy plateau because the backscattering which produces the high energy electrons is most strongly affected by the more attractive short range part of the real potential.

Spectra were also obtained for the ionization of neon by $0.78 \mu \mathrm{m}$ radiation. The ion and electron spectra show an evolution consistent with "pure" tunnel ionization near the saturation intensity. Results for both helium and neon are compiled in Fig. 2 along with calculated curves. The plot shows the ratio of electrons with energies $>2 U_{p}$ over those with energy $<2 U_{p}$ as a function of intensity. The open and filled stars represent the values derived from the experiment for helium and neon respectively. The three theoretical curves are calculated using Coulomb (dashed), $\mathrm{He}^{+}$(solid), and $\mathrm{Ne}^{+}$(dotted) potentials, and include averaging over the laser intensity distribution. The helium data agrees well with the calculated curve (solid line) for the two highest intensities but deviates near the lowest intensity, due to the multiphoton contribution which is not included in our calculation. The neon ratio point (filled star) shows that at the same intensity as helium, neon is approximately ten times more efficient at producing high energy electrons. This result is quite consistent with the observed ten fold increase in the high harmonic emission ${ }^{15}$ for neon over helium. This difference is reflected in the calculated curves and again demonstrates the importance of the short range part of the potential in accurately reproducing the measured results at these intensities. Further evidence is seen in the Coulomb curve, which underestimates the amount of elastic rescattering. However, as the intensity decreases the differences 
1. Total helium PE energy distribution for $0.78 \mu \mathrm{m}$ excitation at (a) 12 , (b) 8 and (c) $4 \times 10^{14}$ $\mathrm{W} / \mathrm{cm}^{2}$. The experimental and calculated distributions using the complete semi-classical theory presented here correspond to the solid and dashed lines, respectively. In panel (b) the dotted line results from pure Coulomb rescattering and the dashed-dotted is without rescattering (SQC model). The Keldysh parameter, $\gamma$, is indicated for each intensity. 
2. Compiled experimental and calculated ratio of total number of electrons with energies $>2 U_{p}$ over those $<2 U_{p}$.

between all three curves vanish and in fact approach the Coulomb scattering result. Since the electron's return energy falls linearly with intensity, collisions with the core are softer, consequently diminishing the influence of the short range physics. Of course, experimental access to this regime is impossible due to the overwhelming contribution of MPI $(\gamma>1)$.

\section{DOUBLE IONIZATION AND THE RESCATTERING MODEL}

Ion yields as a function of intensity were also collected for both helium and neon. Walker et al ${ }^{5}$ have exploited the kilohertz laser technology to extend the range of these measurements by over five orders of magnitude over previous measurements and demonstrate the connection between the nonsequential (NS) double ionization rate and tunneling ionization of the neutral. A sensitive measure of the nonsequential dynamics is provided by plotting the intensity dependence of the $\mathrm{X}^{2+} / \mathrm{X}^{+}$ratio $(\mathrm{X}=$ $\mathrm{He}$ or $\mathrm{Ne}$ ) for both helium (solid circles) and neon (open circles) for $0.78 \mu \mathrm{m}$ excitation, as shown in Fig. 3. To ensure accuracy, the two ions are concurrently collected at a fixed intensity and averaged for at least $10^{6}$ laser shots. The plot shows that the measured NS yield is similar for both atoms, achieving a value of $0.0020[3]$ for helium and $0.0018[4]$ for neon at their saturation intensity. Below saturation, the ratio of each decreases by approximately a factor of 10 over the measured intensity range, although the total rates are changing by seven orders of magnitude. Furthermore, analysis shows that both the helium and neon ratio scale with the tunneling fraction of the ionization, again providing an important clue to the NS dynamics.

The complete quasiclassical calculation, described in Section 2, can be used to predict the double-to-single ionization ratio produced from $\mathrm{e}-2 \mathrm{e}$ inelastic rescattering. Figure 3 shows the results for both helium (solid line) and neon (dashed line). These results are calculated using the same initial conditions and core potentials used to calculate the photoelectron spectra of Figs. 1 and 2. A modified "field-free" e-2e Lotz cross-section ${ }^{13}$ is used to account for double ionization contributions from both core excitation and direct ionization. It has been shown ${ }^{14}$ that the use of field-free cross sections is a reasonable approximation since the slowly varying electric field from 
3. Compiled experimental and calculated ratio of nonsequential double ionization to single ionization for helium and neon for $0.78 \mu \mathrm{m}$ excitation.

the laser has a very small effect on the inelastic scattering processes. Clearly, the $\mathrm{e}-2 \mathrm{e}$ rescattering severely underestimates the absolute measured ratio, as well as the shape. The ratio of the experimental to calculated value at saturation is 47 for helium and 5 for neon. The lack of agreement is a clear indication that more than inelastic rescattering is involved in the physics of the nonsequential ionization. Reasonable assumptions on wave packet spreading and cross sections yield accurate predictions for the electron distributions seen in Fig. 1 and support the estimates used in our model.

The disagreement between the rescattering predictions and the experiment goes beyond underestimating the absolute value. As seen in Fig. 3, the experimental data shows a smooth decrease in the ratio with decreasing intensity, whereas the calculations show a sharp and abrupt cutoff. The origin of the cutoff is clear: as the intensity is lowered, the electron's return energy decreases to the point that it can no longer free the second electron. We see no evidence of this cutoff, even when we repeat the the experiment at $\lambda=0.39 \mu \mathrm{m}$, where all of the NS ionization occurs at intensities below where the cutoff should occur, at a rate (0.0013 time the single ionization rate) very close to that at $0.78 \mu \mathrm{m}$. Additionally, it is difficult to rationalize in a rescattering picture why the double ionization ratios would be the same for helium and neon, considering the difference in the e-2e cross sections. The good agreement found between the complete quasiclassical calculation and the experimental electron distributions demonstrated the important distinction produced by the atom's shortrange potential. Obviously the calculated curves in Fig. 3 reflect the difference in the ionization cross sections, while the experiment does not.

\section{CONCLUSION}

All of the evidence suggests that in the strong-field tunneling limit, the rescattering model captures, even quantitatively, the essential physics leading to the production of high energy electrons and harmonics. However, e-2e rescattering fails, even 
qualitatively, to reproduce most of the salient features of the experiment. We propose that strong-field double ionization is dominated by some other mechanism involving simultaneous two-electron ejection either through a shake-off ${ }^{16}$ or threshold mechanism. While the underlying mechanism for this process remains unclear, various theoretical two-electron ${ }^{17,18}$ treatments have begun. The results we have presented better define the underlying dynamics of these processes and provide more stringent limits on any future models.

\section{ACKNOWLEDGMENTS}

This research was carried out in part at Brookhaven National Laboratory under contract No. DE-AC02-76CH00016 with the U.S. Department of Energy and supported by its Division of Chemical Sciences, Office of Basic Energy Sciences, and in part under the auspices of the U. S. Department of Energy at the Lawrence Livermore National Laboratory under contract No. W-7405-ENG-48. L. F. D. and P. A. acknowledge travel support from NATO under Contract No. SA.5-2-05(RG910678).

\section{REFERENCES}

1. For a recent review, see DiMauro L F and Agostini P 1995 Advances in Atomic, Molecular, and Optical Physics 35 Bederson B and Walther H, Eds. (San Diego: Academic Press)

2. Keldysh L V 1965 Sov. Phys. JETP $201307-1311$

3. Corkum P B, Burnett N H and Brunel F 1989 Phys. Rev. Lett. 62 1259-1262

4. Mohideen U, Sher M H, Tom H W K, Aumiller G D, Wood II O R, Freeman R R, Bokor J and Bucksbaum P H 1993 Phys. Rev. Lett. 71 509-512

5. Walker B, Sheehy B, DiMauro L F, Agostini P, Schafer K J and Kulander K C 1994 Phys. Rev. Lett. 73 1227-1230

6. Yang B, Schafer K J, Walker B, Kulander K C, Agostini P and DiMauro L F 1993 Phys. Rev. Lett. $713770-3773$

7. Paulus G G, Becker W, Nicklich W and Walther H 1994 J. Phys. B: At. Mol. Phys. 27 L703-L708

8. Schafer K J, Yang B, DiMauro L F and Kulander K C 1993 Phys. Rev. Lett. 70 1599-1602

9. Corkum P B 1993 Phys. Rev. Lett. 71 1994-1997

10. Lewenstein M, Kulander K C, Schafer K J and Bucksbaum P H 1995 Phys. Rev. A51 1495-1507

11. Schiff L I 1968 Quantum Mechanics (New York: McGraw Hill)

12. Ammosov M V, Delone N B and Krainov V P 1986 Sov. Phys. JETP 64 1191-1997

13. Lotz W 1968 Z. Phys. 216 241-247

14. Kulander K C, Cooper J and Schafer K J 1995 Phys. Rev. A51 561-568

15. L'Huillier A and Balcou P 1993 Phys. Rev. Lett. 70 774-777

16. Fittinghoff D, Bolton P R, Chang B and Kulander K C 1992 Phys. Rev. Lett. 69 2642-2645

17. Becker A and Faisal F H M 1996 J. Phys. B: At. Mol. Phys. 29 L197-L202

18. Watson J B, Sanpera A, Lappas D G, Knight P L and Burnett K 1996 Proceedings of ICOMP VII (Bristol: IOP) 


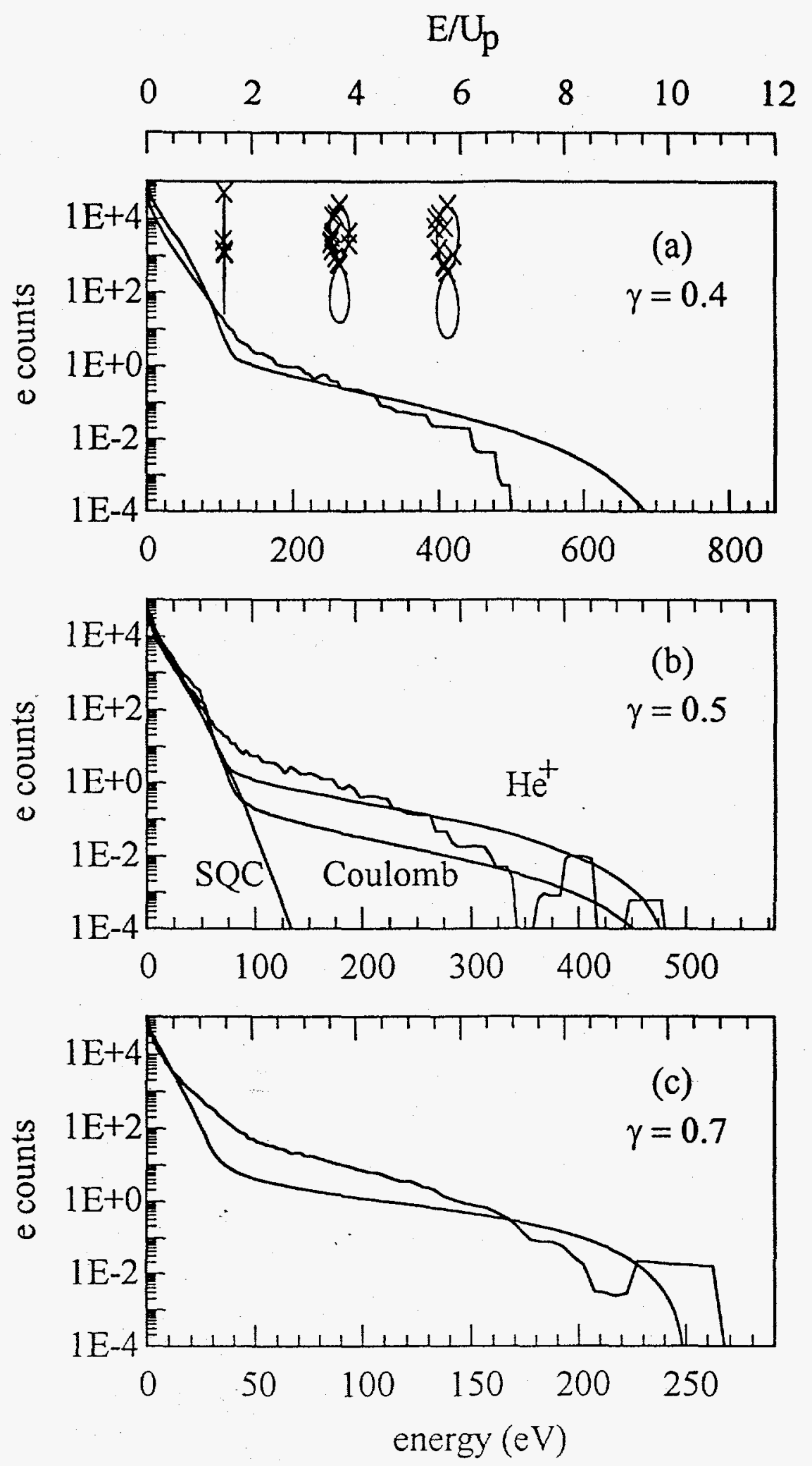

Figure 1: B. Sheehy et al 


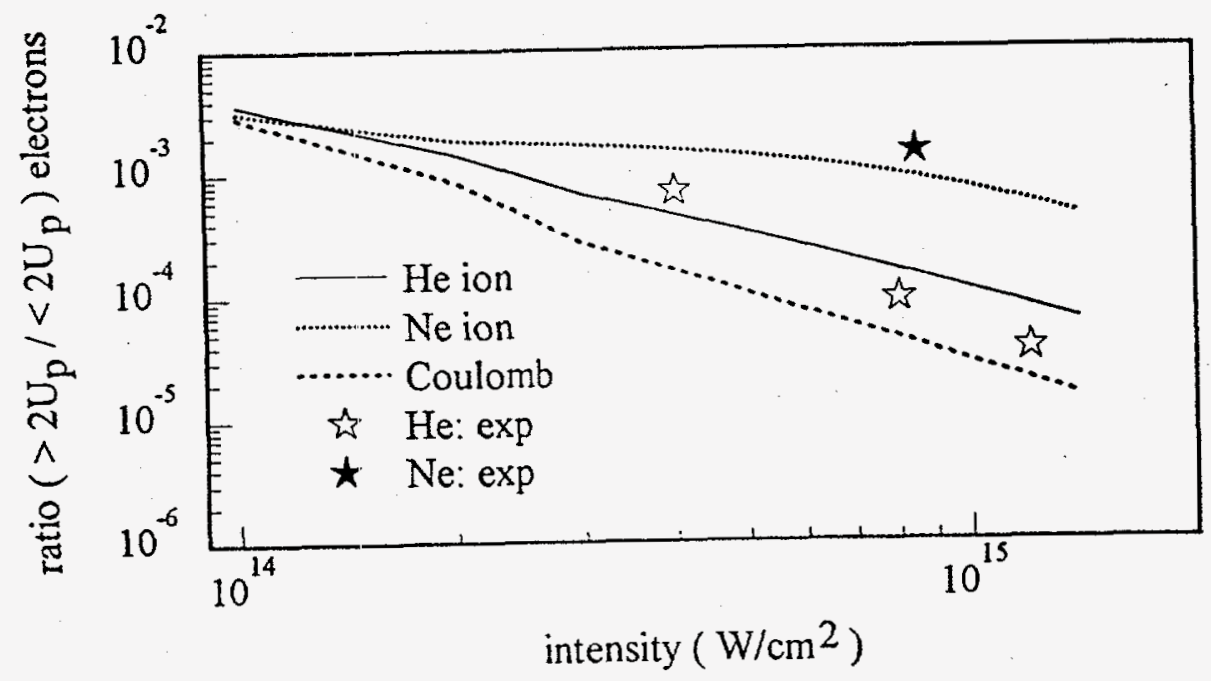

Figure 2: B. Sheehy et al. 


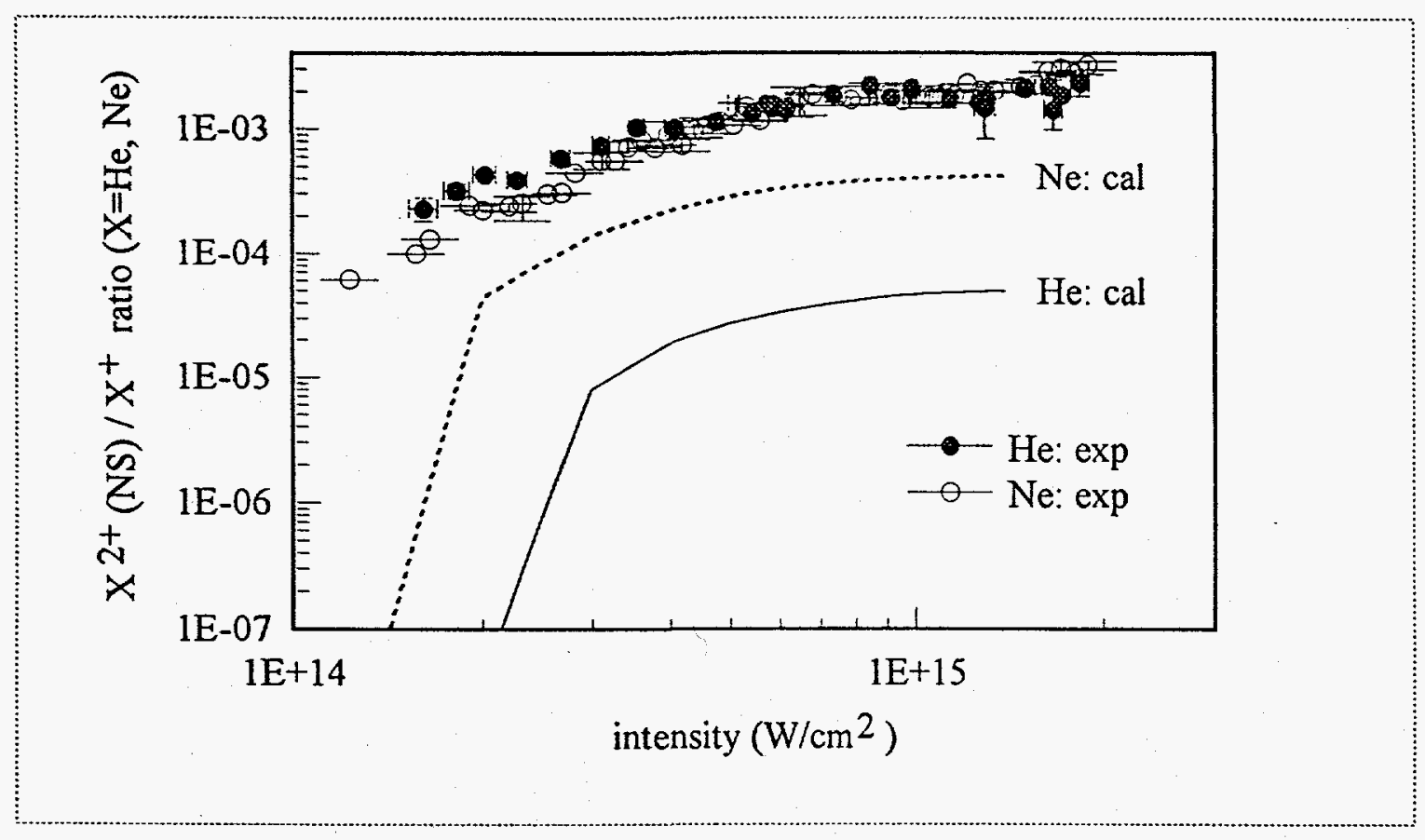

Figure 3: B. Sheehy et al. 